\title{
THE MATHEMATICAL MODEL OF OPTICAL REMOTE SENSING SYSTEM SIGNAL CONSIDERING BROKEN CLOUDINESS EFFECTS
}

\author{
V.P. Budak, O.V. Shagalov* \\ Moscow Power Engineering University, Light Engineering Department, Moscow, 111250 Krasnokasarmennaya Street 14, Russian \\ Federation -ShagalovOV@gmail.com
}

KEY WORDS: Broken clouds, Synthetic iterations, Quasi two-stream approximation

\begin{abstract}
:
With increasing of the accuracy of measuring equipment for the optical remote sensing in recent years the requirements for speed and accuracy of the algorithms for satellite data processing has greatly increased. It became necessary accurately to account all of the known factors, which affect the signal significantly. At each time, more than half of the planet is covered with clouds, so it is almost always necessary to take measurements into breaks in clouds. Cloudiness is among those factors which affect significantly the signal and its neglect in extreme cases can lead to an error of $140 \%$. Here we propose a new solution of the radiative transfer equation (RTE) for a slab of a turbid medium with consideration of broken clouds. We use the classical approach to solving RTE: complete solution is represented as the sum of the anisotropic and regular parts. We express anisotropic part using small-angle modification of the spherical harmonics method. For the regular part we propose to use quasi two-stream approximation. This method is a special case of the synthetic iterations method. The method is based on splitting the ordinary iteration into two stages. At the first step one of approximate methods is used, and on the second step one ordinary iteration is used. We use two-stream approximation as an approximate method. In this paper we proposed a solution for the simplest case of broken clouds - cylindrical hole in the slab. Comparison of the algorithm was performed with established program MDOM, and showed good agreement.
\end{abstract}

\section{INTODUCTION}

\subsection{Problem description}

In recent years measuring equipment for the optical remote sensing had made a significant step forward as far as measurement accuracy is concerned. Thereby now it is possible to face one of the most actual humanity's challenge associated with the threat of global warming: to provide observations of atmospheric small gaseous components with necessary accuracy (Rayner and O'Brien, 2001; Suto et al., 2008). In turn it led to increasing of the requirements for speed and accuracy of the algorithms for satellite data processing. It became necessary to account accurately all of the known factors, which affect the signal significantly, including polarization, anisotropy of scattering, terrain reflection, and true absorption. Among these factors also are effects due to broken cloudiness. Cloudiness affects significantly the signal and its neglect in extreme cases can lead to an error up to $140 \%$ (Kassianov et al., 2009).

\subsection{Proposed solution}

In current paper we propose a new solution of the RTEp for a slab of a turbid medium with consideration of broken clouds, based on quasi two-stream approximation. We use classical representation of the full RTE solution: as a sum of anisotropic part (which comprises all singularities and represented analytically) and regular part (which is a smooth function and calculated numerically). For anisotropic part computations we use small-angle modification of the spherical harmonics method (MSH). For the regular part we use quasi two-stream approximation. This method is a special case of the synthetic iterations method proposed in nuclear physics (Adams and
Larsen, 2002). Now we apply it in radiative transfer theory. It based on a combination of approximate method and one ordinary iteration. As the broken cloudiness case we consider the simplest situation when we have the cylindrical hole in the cloud slab. For this case we use diffusion approximation instead of two-stream approximation because it is more convenient for the arbitrary geometry generalization. After that we use the hexagonal mesh to calculate the first iteration.

\section{QUASI TWO-STREAM APPROXIMATION}

\subsection{Boundary value problem of RTE for a homogeneous} slab

We consider the case, where the slab with optical depth $\tau_{0}$ is irradiated by flat unidirectional source with angle of incidence $\theta_{0}$. Boundary value problem of RTE in this case is

$\left\{\begin{array}{l}\mu \frac{d L(\tau, \mu, \varphi)}{d \tau}=-L(\tau, \mu, \varphi)+\frac{\Lambda}{4 \pi} \oint x\left(\hat{\mathbf{l}}, \hat{\mathbf{I}}^{\prime}\right) L\left(\tau, \mu^{\prime}, \varphi^{\prime}\right), \\ \left.L(\tau, \mu, \varphi)\right|_{\tau=0, \mu \geq 0}=\delta\left(\mu-\mu_{0}\right),\left.L(\tau, \mu, \varphi)\right|_{\tau=0, \mu \geq 0}=0,\end{array}\right.$

where $L(\tau, \mu, \varphi)$ is the radiance of the light field at the optical depth $\tau$, in direction which is defined by $\mu=\cos \theta$ and $\varphi(\theta$ and $\varphi$ are zenith and azimuth angles accordingly), $\mu_{0}=\cos \theta_{0}, \Lambda$ is the single scattering albedo, $x\left(\hat{\mathbf{I}}^{\prime}, \hat{\mathbf{l}}\right)$ is the phase function (here and after unit vectors are marked with symbol “ ” ”). The integration in equation (1) is by full solid angle $4 \pi$, and $d \hat{\mathbf{l}}^{\prime}$ is an elementary solid angle in the direction $\hat{\mathbf{l}}^{\prime}$.

* Corresponding author 


\subsection{Anisotropic part solution}

The base of the RTE solution is to substitute the scattering integral by finite sum with aim to use later one or another numerical method. But there is a $\delta$-singularity in boundary conditions which requires infinite number of decomposition terms. So let us represent the full solution as a sum

$$
L(\mathbf{r}, \hat{\mathbf{l}})=L_{a}(\mathbf{r}, \hat{\mathbf{l}})+\tilde{L}(\mathbf{r}, \hat{\mathbf{l}}),
$$

where $L_{a}(\mathbf{r}, \hat{\mathbf{l}})$ is the anisotropic part (comprises the singularity) and $\tilde{L}(\mathbf{r}, \hat{\mathbf{l}})$ is the regular (smooth) part of the solution. For computation of the anisotropic part of the solution we use MSH. This method based on the radiance angle distribution expanding in series by spherical functions taking into account the following property. The function, which changes fast according to its argument in spatial range, changes slow according to the number of decomposition term in spectral range. This procedure is described in detail, e.g. in the paper (Budak et al, 2011). So we will not elaborate on this, but only give the final expression:

$$
\begin{aligned}
L_{a}(\tau, \mu, \varphi) & = \\
& =\sum_{k=0}^{\infty} \sum_{m=-k}^{k} \frac{2 k+1}{4 \pi} Z_{k}(\tau) \mathrm{Q}_{k}^{n}\left(\mu_{0}\right) \mathrm{Q}_{k}^{m}(\mu) \mathrm{e}^{i m \varphi},
\end{aligned}
$$

where

$$
Z_{k}(\tau)=\exp \left[-\frac{\left(1-\Lambda x_{k}\right) \tau}{\mu_{0}}\right]
$$

$x_{k}$ is the phase function decomposition term, $\mathrm{Q}_{k}^{n}$ is the renormalized Legendre polynomial. It should be noted that the way of representation of the anisotropic part (from the point of view of expressions) is only affects on the source function form, but from the point of view of the full solution MSH is the best method to eliminate the anisotropic part of the solution (Budak and Fedosov, 1985). It allows obtaining the regular part of the solution like a very smooth, almost isotropic function.

\subsection{Regular part solution}

If we substitute the sum (2) in the initial boundary value problem we obtain a new equation for the regular part of the solution

$$
\begin{aligned}
\mu \frac{\partial \tilde{L}(\tau, \mu, \varphi)}{\partial \tau} & +\tilde{L}(\tau, \mu, \varphi)= \\
= & \frac{\Lambda}{4 \pi} \oint x\left(\hat{\mathbf{l}^{\prime}}, \hat{\mathbf{l}}\right) \tilde{L}\left(\tau, \mu^{\prime}, \varphi^{\prime}\right) d \hat{\mathbf{l}}^{\prime}+Q\left(\tau, \mu, \mu^{\prime}, \varphi, \varphi^{\prime}\right),
\end{aligned}
$$

where

$$
\begin{aligned}
Q\left(\tau, \mu, \mu^{\prime}, \varphi, \varphi^{\prime}\right)= & \frac{\Lambda}{4 \pi} \oint x\left(\hat{\mathbf{l}}, \hat{\mathbf{l}}^{\prime}\right) L_{a}\left(\tau, \mu^{\prime}, \varphi^{\prime}\right) d \hat{\mathbf{l}}^{\prime} \\
& -\mu \frac{\partial L_{a}(\tau, \mu, \varphi)}{\partial \tau}-L_{a}(\tau, \mu, \varphi)
\end{aligned}
$$

is the source function which is a misclosure due to anisotropic part of the solution. For the regular part of the solution in this case we use quasi two-stream approximation, which is the combination of two-stream approximation and one ordinary iteration. Two stream-approximation method is based on RTE integration by upper and lower hemispheres. This approach is the simplest method for RTE solution so it seems to be the fastest from computer implementation's point of view. After integration we have two ordinary non-homogeneous differential equations for the hemi-spherical irradiancies (in matrix form):

$$
\overleftrightarrow{\mathrm{M}} \frac{d \tilde{\mathrm{E}}(\tau)}{d \tau}=-\ddot{\mathrm{A}} \tilde{\mathrm{E}}(\tau)+\Lambda e^{-\tau / \mu_{0}} \overrightarrow{\mathrm{F}}
$$

where $\tilde{\mathrm{E}}(\tau)$ is the vector-column of the hemi-spherical irradiancies, $\overrightarrow{\mathrm{M}}$ is the diagonal matrix which contains average scattering cosines, $\vec{A}$ is the square system matrix and $\vec{F}$ is the term which defined by the method of anisotropic part elimination. We use the representation of matrix exponent with eigen vectors and eigen values and obtain the solution of this equation:

$$
\begin{aligned}
\tilde{\mathrm{E}}(\tau)=e^{-\ddot{\mathrm{B}} \tau} \tilde{\mathrm{E}}(0)+ & \\
& +\Lambda \mu_{0} \mathrm{e}^{-\ddot{\mathrm{B}} \tau}\left(\overrightarrow{1}-\mu_{0} \overrightarrow{\mathrm{B}}\right)^{-1}\left(\overrightarrow{1}-\mathrm{e}^{-\tau / \mu_{0}} \mathrm{e}^{\ddot{\mathrm{B}} \tau}\right) \overrightarrow{\mathrm{M}}^{-1} \overrightarrow{\mathrm{F}},
\end{aligned}
$$

where $\overrightarrow{\mathrm{B}}=\overrightarrow{\mathrm{M}}^{-1} \overrightarrow{\mathrm{A}}$. After this we can evaluate the scattering integral in the equation (5) and obtain the radiance of the first iteration of the smooth part of the solution

$$
\begin{gathered}
\left.\tilde{L}\left(\tau_{0}, \mu\right)\right|_{\mu>0}=\frac{\mathrm{e}^{-\tau_{0} / \mu}}{\mu} \int_{0}^{\tau_{0}} \mathrm{e}^{\tau / \mu}\left[W_{\downarrow}(\tau)+\frac{\Lambda}{4 \pi} x\left(\hat{\mathbf{l}}_{0}, \hat{\mathbf{l}}\right) \mathrm{e}^{-\tau / \mu_{0}}\right] d \tau, \\
\left.\tilde{L}(0, \mu)\right|_{\mu<0}=-\frac{1}{\mu} \int_{0}^{\tau_{0}} \mathrm{e}^{\tau / \mu}\left[W_{\uparrow}(\tau)+\frac{\Lambda}{4 \pi} x\left(\hat{\mathbf{l}}_{0}, \hat{\mathbf{l}}\right) \mathrm{e}^{-\tau / \mu_{0}}\right] d \tau,
\end{gathered}
$$

where $W_{\downarrow}(\tau)$ and $W_{\uparrow}(\tau)$ are linear combinations of the hemispherical irradiancies and scattering coefficients.

\section{CLOUD SLAB}

\subsection{Boundary value problem of RTE for the cylindrical hole in the cloud slab}

Let us consider the case, where the cloud slab with cylindrical hole is irradiated by flat unidirectional source with angle of incidence $\theta_{0}$ (geometry is presented on Figure 1).

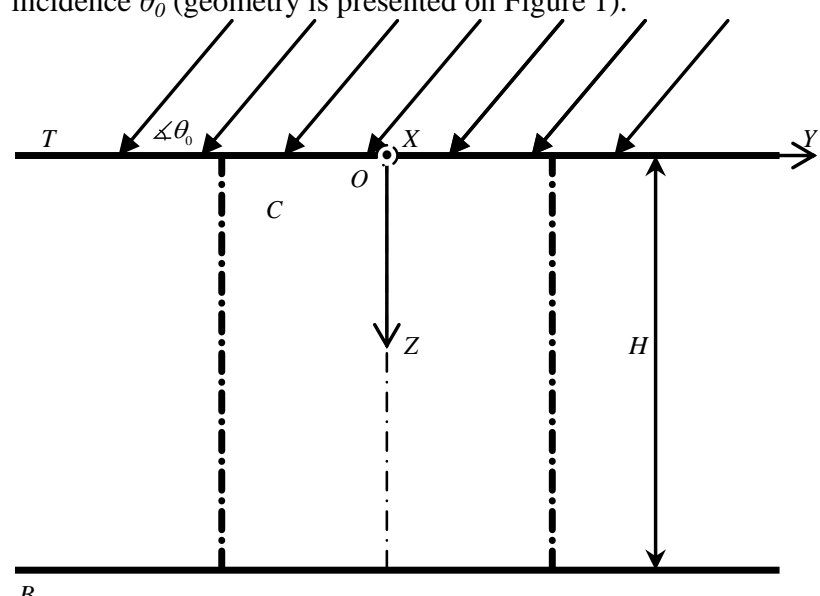

Figure 1. Geometry and nomenclature in case of the cylindrical hole in the turbid medium 
Boundary value problem of RTE in this case is:

$$
\left\{\begin{array}{l}
(\hat{\mathbf{l}}, \nabla) L(\mathbf{r}, \hat{\mathbf{l}})= \\
-\varepsilon(\mathbf{r}) L(\mathbf{r}, \hat{\mathbf{l}})+\frac{\Lambda(\mathbf{r}) \varepsilon(\mathbf{r})}{4 \pi} \oint L\left(\mathbf{r}, \hat{\mathbf{l}}^{\prime}\right) x\left(\mathbf{r} ; \hat{\mathbf{l}}^{\prime}, \hat{\mathbf{l}}\right) d \hat{\mathbf{l}}^{\prime}, \\
\left.L(\mathbf{r}, \hat{\mathbf{l}})\right|_{\mathbf{r} \in T, \hat{\mathbf{l}} \in \Omega_{+}}=\delta\left(\hat{\mathbf{l}}-\hat{\mathbf{l}}_{0}\right),\left.L(\mathbf{r}, \hat{\mathbf{l}})\right|_{\mathbf{r} \in B, \hat{\mathbf{l}} \in \Omega_{-}}=0,
\end{array}\right.
$$

where $L(\mathbf{r}, \hat{\mathbf{l}})$ is radiance of the light field in point $\mathbf{r}$, in direction $\hat{\mathbf{l}}, \varepsilon(\mathbf{r})$ is the attenuation coefficient, the sum of the scattering and absorption coefficients, $\mathrm{T}$ and $\mathrm{B}$ are upper and lower boundaries of the slab accordingly, $\mathrm{C}$ is the cylindrical hole in the slab, $\mathrm{H}$ is the height of the slab. Origin of coordinates OXYZ is in the centre of upper base of the cylindrical hole. The parameters of the cloud slab are

$$
\varepsilon(\mathbf{r}), \Lambda(\mathbf{r}), x\left(\mathbf{r} ; \hat{\mathbf{l}}^{\prime}, \hat{\mathbf{l}}\right)=\left\{\begin{array}{c}
\varepsilon, \Lambda, x\left(\hat{\mathbf{l}}^{\prime}, \hat{\mathbf{l}}\right), \quad \mathbf{r} \notin C, \\
0, \mathbf{r} \in C .
\end{array}\right.
$$

\subsection{Anisotropic part}

Anisotropy is a local property of the equation, so for its definition we assume approximation of independent slab of turbid medium with optical thickness equals to the optical thickness for direct rays. Due to this factor the expression for the anisotropic part will be same as (3) but with optical thickness $\xi(\mathbf{r})$ which is depends on spatial coordinates.

\subsection{Optical thickness computation}

We have to find the intersection of the ray and cylinder with aim to obtain the expression for the optical thickness:

$$
\mathbf{r}=\mathbf{d}+\xi \hat{\mathbf{l}}_{0}
$$

is equation of the ray along the direction of the incidence of the unidirectional source, $\mathbf{d}$ is the vector of ray's input of the independent slab;

$$
(\mathbf{r}-(\mathbf{r}, \hat{\mathbf{k}}) \mathbf{k})^{2}=R^{2}
$$

is the point on the cylinder's surface on the distance R from its axis. Let us to substitute (13) in (14)

$$
\xi^{2}+2\left(\mathbf{d}-(\mathbf{r}, \hat{\mathbf{k}}) \mathbf{k}, \hat{\mathbf{l}}_{0}\right)+(\mathbf{d}-(\mathbf{r}, \hat{\mathbf{k}}) \mathbf{k})^{2}-R^{2}=0 .
$$

Now we have the second-degree equation and can obtain an optical thickness $\xi$. If the ray does not cross the cylindrical hole $\xi=H / \mu_{0}$.

\subsection{Regular part solution}

We also substitute the radiance in the initial boundary value problem by the sum (2) and obtain a new equation for the regular part of the solution

$$
(\hat{\mathbf{l}}, \nabla) \tilde{L}(\mathbf{r}, \hat{\mathbf{l}})+\varepsilon \tilde{L}(\mathbf{r}, \hat{\mathbf{l}})=\frac{\Lambda \varepsilon}{4 \pi} \oint \tilde{L}\left(\mathbf{r}, \hat{\mathbf{I}}^{\prime}\right) x\left(\hat{\mathbf{I}}^{\prime}, \hat{\mathbf{l}}\right) d \hat{\mathbf{l}}^{\prime}+S(\mathbf{r}, \hat{\mathbf{l}})
$$

$$
\begin{aligned}
S(\mathbf{r}, \hat{\mathbf{l}})=\frac{\Lambda \varepsilon}{4 \pi} \oint L_{a}\left(\mathbf{r}, \hat{\mathbf{l}}^{\prime}\right) x\left(\hat{\mathbf{I}}^{\prime}, \hat{\mathbf{l}}\right) d \hat{\mathbf{l}}^{\prime}- & \\
& -(\hat{\mathbf{l}}, \nabla) L_{a}(\mathbf{r}, \hat{\mathbf{l}})-\varepsilon L_{a}(\mathbf{r}, \hat{\mathbf{l}})
\end{aligned}
$$

In case of the cylindrical hole we deal with changing of the axis of the symmetry respect to two-stream approximation. Especially it becomes important in case of arbitrary geometry. So this approach is not appropriate here. The next one after it from the implementation simplicity's point of view seems to be diffusion approximation. Thereby for the first step in the method of synthetic iterations for this case we suggest to use diffusion approximation instead of two-stream approximation. Further it will be more convenient to generalize it on arbitrary geometry. Diffusion approximation is based on the decomposition of the radiance angle distribution in the series by spherical harmonics with saving of the first two terms:

$$
\begin{aligned}
& L(\mathbf{r}, \hat{\mathbf{l}})=C_{00}(\mathbf{r}) \mathrm{Y}_{0}^{0}(\hat{\mathbf{l}})+\sum_{m=-1}^{1} C_{1 m}(\mathbf{r}) \mathrm{Y}_{1}^{m}(\hat{\mathbf{l}})= \\
& =\frac{1}{4 \pi} E_{0}(\mathbf{r})+\frac{3}{4 \pi} \mathcal{E}(\mathbf{r}) \hat{\mathbf{l}}=\frac{1}{4 \pi}\left(E_{0}(\mathbf{r})+3 \mathcal{E}(\mathbf{r}) \hat{\mathbf{l}}\right) \\
& S(\mathbf{r}, \hat{\mathbf{l}})=s_{00}(\mathbf{r}) \mathrm{Y}_{0}^{0}(\hat{\mathbf{l}})+\sum_{m=-1}^{1} s_{1 m}(\mathbf{r}) \mathrm{Y}_{1}^{m}(\hat{\mathbf{l}}) \equiv \\
& \equiv \frac{1}{4 \pi}\left(s_{0}(\mathbf{r})+3 \mathbf{s}(\mathbf{r}) \hat{\mathbf{l}}\right), \\
& x\left(\hat{\mathbf{l}}^{\prime} \cdot \hat{\mathbf{l}}\right)=\sum_{l=0}^{\infty} \frac{21+1}{4 \pi} x_{l} \mathrm{P}_{l}\left(\hat{\mathbf{l}^{\prime}} \cdot \hat{\mathbf{l}}\right)
\end{aligned}
$$

where

$$
\begin{aligned}
& E_{0}(\mathbf{r})=\oint L(\mathbf{r}, \hat{\mathbf{l}}) d \hat{\mathbf{l}}, \quad \mathcal{E}(\mathbf{r})=\oint L(\mathbf{r}, \hat{\mathbf{l}}) \hat{\mathbf{l}} d \hat{\mathbf{l}}, \\
& s_{0}(\mathbf{r})=\oint S(\mathbf{r}, \hat{\mathbf{l}}) d \hat{\mathbf{l}}, \quad \mathbf{s}(\mathbf{r})=\oint S(\mathbf{r}, \hat{\mathbf{l}}) \hat{\mathbf{l}} d \hat{\mathbf{l}}
\end{aligned}
$$

We substitute (18)-(20) in the (16), consider that

$$
\frac{1}{4 \pi} \oint x\left(\hat{\mathbf{l}}^{\prime}, \hat{\mathbf{l}}\right) d \hat{\mathbf{l}}^{\prime}=1
$$

$$
\begin{aligned}
& \frac{1}{4 \pi} \oint \hat{\mathbf{I}}^{\prime} x\left(\hat{\mathbf{I}}^{\prime}, \hat{\mathbf{l}}\right) d \hat{\mathbf{l}}^{\prime}= \\
& =\frac{1}{4 \pi} \oint\left(\hat{\mathbf{l}}_{\perp} \sqrt{1-\mu^{2}} \cos \varphi+\hat{\mathbf{l}} \mu\right) x\left(\hat{\mathbf{l}}^{\prime}, \hat{\mathbf{l}}\right) d \hat{\mathbf{l}}^{\prime}= \\
& =\frac{1}{4 \pi} \hat{\mathbf{l}} \oint \mu x\left(\hat{\mathbf{l}}^{\prime}, \hat{\mathbf{l}}\right) d \hat{\mathbf{l}}^{\prime}=\hat{\mathbf{l}} \bar{\mu},
\end{aligned}
$$

where $\quad \hat{\mathbf{l}}^{\prime}=\hat{\mathbf{l}}_{\perp} \sqrt{1-\mu^{2}} \cos \varphi+\mu \hat{\mathbf{l}}, \quad \bar{\mu}=\frac{1}{4 \pi} \oint\left(\hat{\mathbf{l}}^{\prime}, \hat{\mathbf{l}}\right) x\left(\hat{\mathbf{l}}^{\prime}, \hat{\mathbf{l}}\right) d \hat{\mathbf{l}}^{\prime}=\frac{x_{1}}{3}$ is the average scattering cosine and obtain

$$
\begin{aligned}
& {[(\hat{\mathbf{l}}, \nabla)+\varepsilon}-\Lambda \varepsilon] E_{0}(\mathbf{r})+ \\
&+3[(\hat{\mathbf{l}}, \nabla)+\varepsilon-\Lambda \varepsilon \bar{\mu}] \hat{\mathbf{l}} \mathcal{E}(\mathbf{r})=s_{0}(\mathbf{r})+3 \mathbf{s}(\mathbf{r}) \hat{\mathbf{l}} .
\end{aligned}
$$

where source function is 
Let us integrate obtained expression by solid angle, taking into account that

$$
\begin{aligned}
& \oint \hat{\mathbf{l}} d \hat{\mathbf{l}}=0, \\
& \oint \hat{\mathbf{l}} \mathcal{E}(\mathbf{r}) d \hat{\mathbf{l}}=\mathcal{E}(\mathbf{r}) \oint \hat{\mathbf{l}} d \hat{\mathbf{l}}=0, \\
& \oint(\hat{\mathbf{l}}, \nabla) \hat{\mathbf{l}} \mathcal{E}(\mathbf{r}) d \hat{\mathbf{l}}=\nabla \oint \hat{\mathbf{l}} \hat{\mathbf{l}} \mathcal{E}(\mathbf{r})) d \hat{\mathbf{l}}=\frac{4 \pi}{3} \nabla \mathcal{E}(\mathbf{r}),
\end{aligned}
$$

that lead to the following expression:

$$
\varepsilon(1-\Lambda) E_{0}(\mathbf{r})+\nabla \mathcal{E}(\mathbf{r})=s_{0}(\mathbf{r})
$$

We multiply equation (24) by $\hat{\mathbf{I}}$ and integrate it by solid angle again:

$$
\frac{1}{3} \nabla E_{0}(\mathbf{r})+\varepsilon(1-\Lambda \bar{\mu}) \mathcal{E}(\mathbf{r})=\mathbf{s}(\mathbf{r})
$$

We assume that the radiance angle distribution due to anisotropic part misclosure is too close to the isotropic one, that

$$
\mathbf{s}(\mathbf{r}) \approx 0
$$

This means that equation (27) can be represented in the following form

$$
\begin{aligned}
& \frac{1}{3} \nabla E_{0}(\mathbf{r})+\varepsilon(1-\Lambda \bar{\mu}) \mathcal{E}(\mathbf{r})=0 \Rightarrow \\
& \Rightarrow \mathcal{E}(\mathbf{r})=-\frac{1}{3} \frac{1}{\varepsilon(1-\Lambda \bar{\mu})} \nabla E_{0}(\mathbf{r}) \equiv D \nabla E_{0}(\mathbf{r}),
\end{aligned}
$$

and it's become possible to lead the equation for the regular part of the solution to diffusion equation

$$
D \Delta E_{0}(\mathbf{r})+\varepsilon(1-\Lambda) E_{0}(\mathbf{r})=s_{0}(\mathbf{r}) .
$$

\subsection{Iteration in case of the cylindrical hole in the slab}

To take the first iteration we have to move to Peierls' integral RTE. To do this we introduce the new coordinate system along the ray (Budak et al., 2011). In this case arbitrary point $\mathbf{r}$ in Cartesian coordinate system from the point $\mathbf{R}$ at the distance $\zeta$ in direction $\hat{\mathbf{l}}$ is defined by expression

$$
\mathbf{r}=\mathbf{R}+\zeta \hat{\mathbf{1}}
$$

We substitute this expression in the RTE and after some transformations obtain

$$
\begin{aligned}
\tilde{L}(\mathbf{R}+\zeta \hat{\mathbf{l}}) & =L_{0}(\mathbf{R}+\zeta \hat{\mathbf{l}})+ \\
& +\frac{\Lambda \varepsilon}{4 \pi} \int_{0}^{\zeta} \mathrm{e}^{\varepsilon\left(v-\zeta_{0}\right)} \oint x\left(\hat{\mathbf{l}}, \hat{\mathbf{l}}^{\prime}\right) \tilde{L}\left(\mathbf{R}+v \hat{\mathbf{l}}^{\prime}, \hat{\mathbf{1}}^{\prime}\right) d \hat{\mathbf{l}}^{\prime} d v
\end{aligned}
$$

where $L_{0}(\mathbf{R}+\zeta \hat{\mathbf{l}})$ is the radiance of direct non-scattering light. To calculate (32) we divide the bulk of medium into the mesh of the hexagonal cells (Mitchell and Wait, 1976), in each node $\mathbf{r}_{\mathrm{ij}}$ of which we store the discrete values of radiance by the fixed direction $\hat{\mathbf{l}}_{p q}$ in accordance with the selected quadrature formula of the integration over the solid angle (Koch and Becker, 2004). At the first step the convolution over the solid angle is calculated in every node. Further the integral convolution values are interpolated between the nodes using one of the approximation schemes while calculating the integral over the ray. Comparison of proposed approach was realized with algorithm, called MDOM (Budak et al., 2011), which uses MSH for the anisotropic part and discrete ordinate method for the regular part of the solution and intrinsically is accurate solution of RTE. MDOM is designed for the case of homogeneous slab so the correct comparison was possible only in case of $R=0$. The results showed that the error for the upper hemisphere is not more than $4 \%$.

\section{CONCLUSIONS}

New method for solving the RTE, consider broken cloudiness effects was proposed. MSH is the best way to eliminate the anisotropic part of the solution which allows obtaining the regular part like almost isotropic function. This factor in its turn allows assuming that it is appropriate to use synthetic iterations method and the simplest method for RTE solving on its first step here. Quasi two-stream approximation is good enough for the homogeneous medium slab, but in case of arbitrary geometry it is more convenient to use diffusion approximation for the first step in synthetic iterations.

\section{REFERENCES}

Adams, M.L., Larsen, E.W., 2002. Fast iterative methods for discrete-ordinates particle transport calculations. Progress in Nuclear Energy, vol. 40, pp. 3-159.

Budak, V.P., Klyuykov, D.A., Korkin, S.V., 2011. Complete matrix solution of radiative transfer equation for pile of horizontaly homogeneous slabs. J. Quant. Spectrosc. Radiat. Transfer, v. 112, pp. 1141-1148.

Budak, V.P., Fedosov, V.P., 1985. On the relation between small-angle approximation forms of the transfer equation. The cycle of matter and energy. in reservoirs, LP SD AS USSR, Irkutsk, pp. 78-79.

Koch, R., Becker, R., 2004. Evaluation of quadrature schemes for the discrete ordinates method. J. Quant. Spectrosc. Radiat. Transf., vol. 84, pp. 423-435.

Mitchell, A.R., Wait, R., 1976. The Finite Element Method in Partial Differential Equation. London: Wiley.

Rayner, P. J., O'Brien, D. M., 2001. The utility of remotely sensed $\mathrm{CO} 2$ concentration data in surface source inversions. Geophysical Research Letters, vol. 1, pp. 175-178.

Suto, H., Kawashima, T., Yoshida, J., Ishida, J., Kuze, A., Nakajima, M., Hamazaki, T., 2008. The pre-launch performance test and calibration results of Thermal And Near infrared Sensor for carbon Observation (TANSO) on GOSAT. Proc. of SPIE, Vol. 7106 71060L, pp. 1-10. 